\title{
Effect of the starting surfaces of GaN on defect formation in epitaxial Co thin films
}

\author{
H. D. Li, ${ }^{1,2}$ T. L. Wong, ${ }^{3}$ N. Wang, ${ }^{3}$ J. Wang, ${ }^{4}$ Q. Li, ${ }^{4}$ and M. H. Xie ${ }^{1, a)}$ \\ ${ }^{1}$ Physics Department, The University of Hong Kong, Pokfulam Road, Hong Kong, People's Republic of China \\ ${ }^{2}$ Department of Physics, Beijing Jiaotong University, Beijing 100044, People's Republic of China \\ ${ }^{3}$ Physics Department, Hong Kong University of Science and Technology, Clear Water Bay, Kowloon, \\ Hong Kong, People's Republic of China \\ ${ }^{4}$ Physics Department, The Chinese University of Hong Kong, Shatin, Hong Kong, People's Republic of China
}

(Received 6 June 2011; accepted 7 September 2011; published online 1 November 2011)

\begin{abstract}
Growths of Co epifilms on $\mathrm{GaN}(0001)-“ 1 \times 1$ " and $(1 \times 1)$ surfaces were studied, where the structural properties of the crystals and the interfaces are compared. Stacking faults are seen to be abundant in epitaxial Co films grown on excess Ga covered GaN(0001)-“ $1 \times 1$ " surface. Such stacking defects are effectively suppressed in Co films grown on less excess Ga covered $\mathrm{GaN}(0001)-(1 \times 1)$ surfaces. The hetero-interface between Co and $\mathrm{GaN}(0001)$ is characterized by a disordered or amorphous region, and diffusion of $\mathrm{Ga}$ and $\mathrm{N}$ from the substrate into Co is suggested. (C) 2011 American Institute of Physics. [doi:10.1063/1.3652761]
\end{abstract}

\section{INTRODUCTION}

Integrating transition metals (TMs) carrying magnetic moments in $\mathrm{GaN}$ has been a subject of extensive research attention in the development of thin film magnetoelectronics $^{1}$ and spintronics based on wide bandgap semiconductors. ${ }^{2}$ To act as an effective spin injector, the integrity of the interface between deposited TMs and GaN is pivotal. The magnetic and electronic properties of the TMs-on-GaN systems will depend sensitively on the structure and chemical compositions of the interfaces. ${ }^{3}$ It is known that interfacial reaction often occurs between TMs and semiconductors, which may lead to complex crystalline phases and interface structures.

Among the most common TM metals, nickel (Ni) and cobalt (Co) show similar close-packed crystal structure to $\mathrm{GaN}$ and are thus considered to be superior candidates for epitaxial TM on GaN. For such heteroepitaxial systems, in addition to the huge chemical difference between TMs and $\mathrm{GaN}$, there exist relatively large lattice mismatches $(\geq 20 \%)$. One important aspect in the epitaxial growth of TM on GaN is thus the effect of surface/interface states on defect formation in epifilms and at the interfaces. In addition, since $\mathrm{GaN}(0001)$ can be prepared into one of many surface states, including excess-Ga covered incommensurate and coherent structures, how such surfaces affect the interface reaction and epitaxial crystal structure is not only of fundamental interests but also important for developing GaN-based magnetoelectronics and spintronics.

Although study of epitaxial $\mathrm{Ni}$ on $\mathrm{GaN}$ has been reported in the literature, ${ }^{4}$ less attention has been paid so far on Co-on-GaN. On the other hand, Co carries a high magnetic moment $\left(\sim 1.7 \mu_{B}\right.$, comparing to $\sim 0.6 \mu_{B}$ of $\mathrm{Ni}$ ) and has the hexagonal close packed (hcp) crystal structure at room temperature (RT), similar to GaN. It is a good candidate worthy of detailed investigation. In a recent effort of molecular-

${ }^{\text {a)} E l e c t r o n i c ~ m a i l: ~ m h x i e @ h k u . h k . ~}$ beam epitaxy (MBE) of $\mathrm{Co}$ on $\mathrm{GaN}$, we have already observed differences in the structural and magnetic properties of epitaxial Co on two different $\mathrm{GaN}(0001)$ substrates: $>2$ monolayers (MLs) excess Ga covered pseudo-" $1 \times 1$ " (denoted by " $1 \times 1$ " hereafter) and a single Ga ML covered $(1 \times 1)$ surface, respectively. Specifically, epitaxial Co films grown on " $1 \times 1$ " contain predominantly domains that are $30^{\circ}$ rotated relative to the lattices of the substrate, but the lattices of Co grown on the $(1 \times 1)$ surfaces are aligned with GaN. ${ }^{5}$ Such a crystal structural difference has led to differences in magnetic properties, such as degraded saturation magnetization in one and in-plane uniaxial anisotropy in the other. ${ }^{5}$ Here, we supplement the previous study and report the distinct differences in defect formation in the two epifilms on $\operatorname{GaN}(0001)$ - " $1 \times 1$ " and $-(1 \times 1)$ surfaces. The structural details of the two interfaces are examined by highresolution transmission electron microscopy (HRTEM), while the compositional information of the epifilms and interfaces are revealed by energy-dispersive X-ray (EDX) analysis and secondary ion mass spectrometry (SIMS) measurements.

\section{EXPERIMENTS}

GaN substrate preparation, Co film deposition and subsequent surface analyses of the samples were carried out in a purpose-built multi-chamber ultrahigh vacuum (UHV) system. It consisted of a radio-frequency (rf) plasma-assisted molecular-beam epitaxy (MBE) reactor for GaN growth and surface preparation, an e-beam deposition chamber for Co growth, and the analysis chambers equipped with reflection high-energy electron diffraction (RHEED), low-energy electron diffraction (LEED), and scanning tunneling microscopy (STM). The rf-MBE system was equipped with a conventional effusion cell of $\mathrm{Ga}$ and a plasma unit for nitrogen $(\mathrm{N})$ flux. The base pressures of all UHV chambers were below $3 \times 10^{-10}$ mbar. The GaN/6 H-SiC(0001) substrates were from TDI, on which a $30 \mathrm{~nm}$-thick GaN buffer was deposited 
prior to surface reconstruction preparations. Co depositions on $\mathrm{GaN}$ were carried out at RT with a typical growth rate of $0.01 \mathrm{MLs} / \mathrm{s}$ (monolayers per second) as measured by a quartz crystal oscillator, and the thickness of the Co films investigated in this study were $\sim 10 \mathrm{~nm}$. Post-growth annealing of the grown sample was conducted by flowing a direct current through the long side of the rectangular sample piece. The temperature was readout from an infrared pyrometer and a thermocouple. Surface morphologies of the grown films were examined by room-temperature STM using the constant-current mode. The sample bias was $+1 \mathrm{~V}$ and the tunneling current was $0.1 \mathrm{nA}$. Surface atomic structures were examined by in situ RHEED and/or LEED operated at $10 \mathrm{keV}$ and $100 \mathrm{eV}$, respectively. Cross-sectional HRTEM characterizations of some samples were done in a highresolution JEOL2010 F microscope, during which EDX spectra were also collected. The TEM specimens were prepared by a standard mechanical thinning and Ar ion milling procedure. SIMS depth profiles were acquired using oxygen or bismuth primary ions in a time-of-flight spectrometer ToF-SIMS V (ION-TOF GmbH).

\section{RESULTS AND DISCUSSIONS}

GaN(0001)-“ $1 \times 1$ " and $(1 \times 1)$ are well established as $>2$ MLs and 1 ML excess Ga covered surfaces, respectively, which can be obtained readily by tuning the $\mathrm{Ga} / \mathrm{N}$ flux ratio during growth and/or post-growth annealing. ${ }^{6}$ The former surface is featured in the RHEED by the satellite streaks close to the $(1,0),(0,0)$, and $(\overline{1}, 0)$ main diffraction streaks, as shown in Fig. 1(a) (pointed by the short black arrows), which results from the incommensurate nature of the topmost excess Ga showing a high surface mobility and thus being fluid-like. This is schematically illustrated at the bottom of Fig. 1(a). ${ }^{7}$ The $(1 \times 1)$ surface is, on the other hand, featured by clean $(1 \times 1)$ RHEED pattern as shown in Fig. 1(b), while detailed analysis of the diffraction data revealed that the surface also contained an excess $\mathrm{Ga}$ adlayer, in which the $\mathrm{Ga}$ adatoms resided coherently at the $\mathrm{T} 1$ sites of the Gaterminated GaN surface [see the schematic drawing below
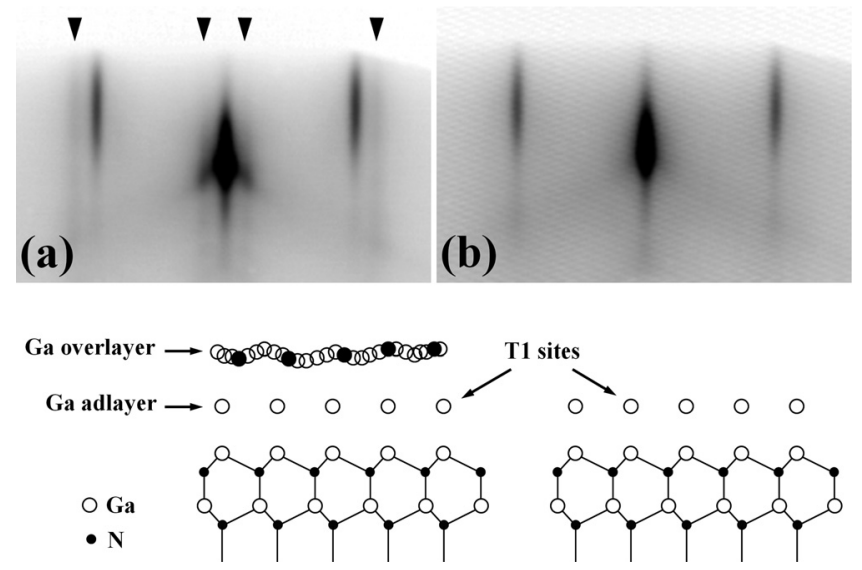

FIG. 1. RHEED patterns taken from (a) $\mathrm{GaN}(0001)-" 1 \times 1$ " and (b) $\mathrm{GaN}(0001)-(1 \times 1)$ substrates in $\langle 11 \overline{1} 0\rangle$ azimuth. Corresponding atomic ball-stick models of $\mathrm{GaN}(0001)-" 1 \times 1$ " and $(1 \times 1)$ are illustrated below their RHEED patterns, respectively (Refs. 7 and 8).
Fig. 1(b)]. ${ }^{8}$ As was revealed earlier, ${ }^{5}$ growth of Co thin film on these two surfaces showed distinguished differences, where on GaN(0001)-" $1 \times 1$ ", the epitaxial film is dominantly $30^{\circ}$ rotated with respect to the lattices of GaN, though domains that are aligned with $\mathrm{GaN}$ are also observable according to the RHEED and LEED. On GaN(0001)- $(1 \times 1)$ surfaces, on the other hand, the grown Co films aligned with GaN lattices without rotation domains. ${ }^{5}$

Besides the orientation difference of the grown crystals, the two substrates also lead to significant differences in surface morphology and defect formation in epitaxial Co. Figures 2(a) and 2(e) show STM micrographs of the asdeposited Co films on GaN(0001)-“ $1 \times 1$ " and $-(1 \times 1)$, respectively. It is seen that the surface in Fig. 2(a) is characterized by the step-and-terrace morphology, signifying the two-dimensional growth mode of the epifilm. However, by examining the details of the morphological features, one observes screw dislocations in the center of the spiral mounds as well as some other defects, such as stacking faults. It also contains face-centered cubic (fcc) domains in the host of a hcp-crystal, an example of which is enclosed by
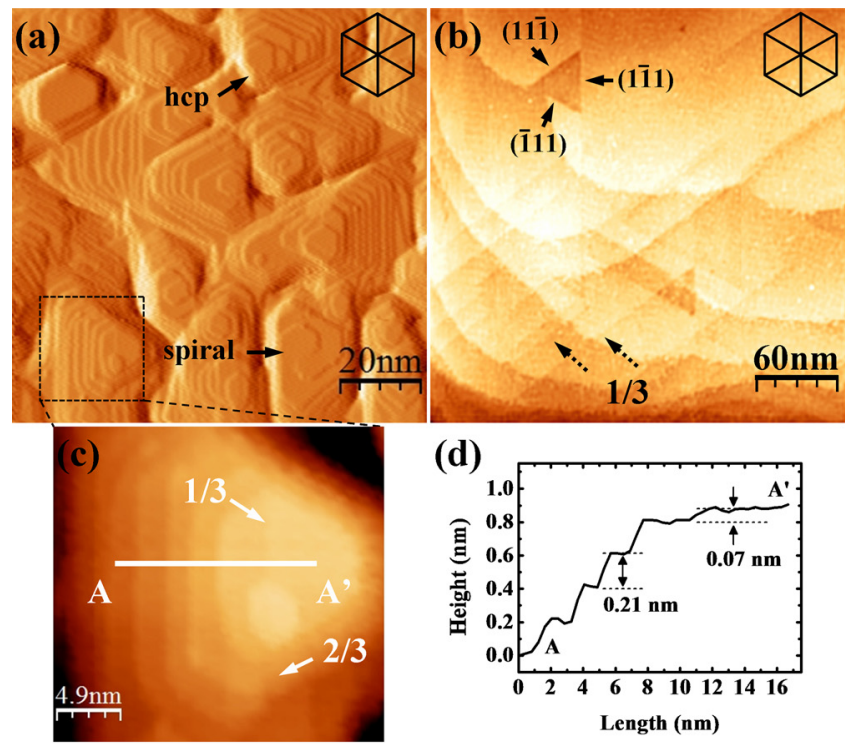

(d)

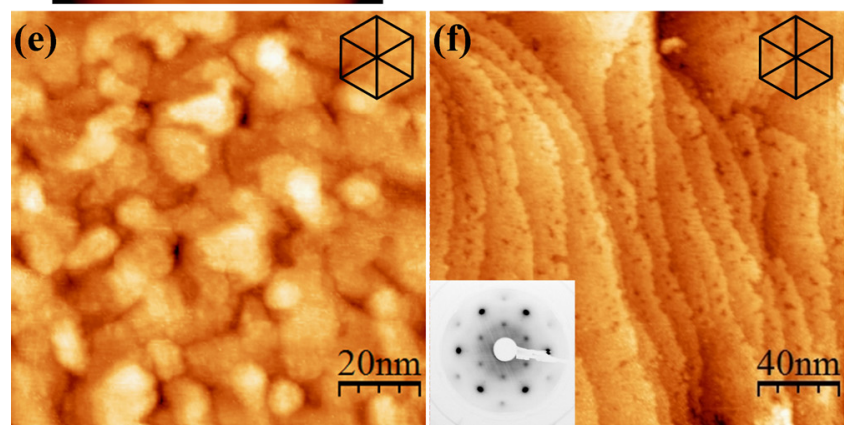

FIG. 2. (Color online) STM images of the as-deposited (a) Co/GaN(0001)-$1 \times 1$ " and (e) $\mathrm{Co} / \mathrm{GaN}(0001)-(1 \times 1)$ samples, respectively. To highlight the step-terrace features, the STM morphology image shown in (a) is a derivative one. Zoom-in image from an area framed by dashed box in (a) is shown in (c). (d) Height profile along AA' in (c), showing a submonolayer step (0.07 nm in height). (b) and (f) are STM images of the annealed Co/ $\mathrm{GaN}(0001)-" 1 \times 1$ " and $\mathrm{Co} / \mathrm{GaN}(0001)-(1 \times 1)$ samples, respectively. The dashed and solid arrows in (b) point to submonolayer high steps and a depression, as caused by stacking faults in film. The LEED pattern shown in the inset of (f) reveals the $(\sqrt{3} \times \sqrt{ } 3)$ reconstruction of the surface. 
a dashed box and magnified as given in Fig. 2(c). The distinction between hcp and fcc domains can be made based on the morphological features, where for the hcp-domain, the spiral mounds are of hexagonal shape and the sides of the mounds contain atomic steps that are twinkled [a typical hcp mound is marked in Fig. 2(a)], while the fcc domain manifests by triangular shaped mounds and parallel steps on the side of the mounds. ${ }^{9}$ Line profile of the mound in Fig. 2(c) reveals submonolayer high steps (i.e., $1 / 3$ or $2 / 3$ of the atomic step height of Co), as shown in Fig. 2(d). These submonolayer steps are fingerprints of stacking faults in bulk fcc crystals intersecting the (111) surface. ${ }^{10}$

It is known that Martensitic phase transition (MPT) of Co from hcp to fcc occurs at elevated temperature $(\sim 700 \mathrm{~K}) .{ }^{11}$ To see more clearly stacking faults in fcc-Co, the film undergoes an annealing procedure at $1000 \mathrm{~K}$ to facilitate the MPT. The resulted film shows a surface morphology that is presented in Fig. 2(b), from which straight and long submonolayer high steps along the $\langle 1 \overline{1} 0\rangle$ directions are abundant. Two such submonolayer high steps are indicated by the dashed arrows in the Fig. 2(b). Three equivalent shear

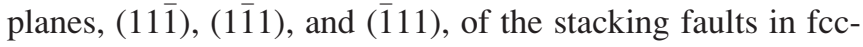
Co may intersect the surface in such a way that triangular depressions are created on surface, as is also depicted in Fig. 2(b) by solid arrows. Therefore, in epitaxial Co films grown on $\mathrm{GaN}(0001)$-“ $1 \times 1$ ”, high density of stacking faults are present, accompanying the rotation domains.

Comparatively, the as-deposited Co films on $\mathrm{GaN}(0001)-(1 \times 1)$ surfaces do not reveal the step-and-terrace morphology [Fig. 2(e)]. It may reflect a lower surface diffusivity of adatoms on the less excess $\mathrm{Ga}$ covered $\mathrm{GaN}(0001)-(1 \times 1)$ surface than on " $1 \times 1$ ". Since such a film contains few rotation domains as described earlier, ${ }^{5}$ which would otherwise facilitate strain relaxation, considerable residual stress may be present in this film leading to more severe surface modulations. Annealing the sample at $1000 \mathrm{~K}$ significantly smoothen the surface, as is apparent from Fig. 2(f). Interestingly, no submonolayer high step is found, signaling few stacking defects in such a film. However, one observes many small pits on the surface of Fig. 2(f), most of which are of triangular shape. We suspect these pits are induced by atom desorption by annealing. Another interesting feature of this surface is the $(\sqrt{3} \times \sqrt{3})$ reconstruction as seen by the LEED [inset of Fig. 2(f)]. Although we do not know the source and identity of this surface superstructure, the $(\sqrt{3} \times \sqrt{3})$ reconstructed surface has previously been noted on $\mathrm{Ni} / \mathrm{GaN}(0001)$ after annealing, which was attributed to out-diffused $\mathrm{Ga}$ from $\mathrm{GaN}$ substrate to the surface of $\mathrm{Ni}(111){ }^{4}$

To examine further the structural details of the epifilms and particularly the interfaces of $\mathrm{Co} / \mathrm{GaN}(0001)-“ 1 \times 1$ " and $\mathrm{Co} / \mathrm{GaN}(0001)-(1 \times 1)$, HRTEM of the samples are conducted and the results are shown in Fig. 3. Firstly, the HRTEM micrographs indeed reveal the fcc stacking of the annealed Co films, confirming the MPT of the epilayers. In fact, by examining over large areas of the TEM specimens, few hcp domains are discernable. However, as the TEM and STM analyses were made at RT, the above observation would also indicate the MPT from fcc back to hep does not
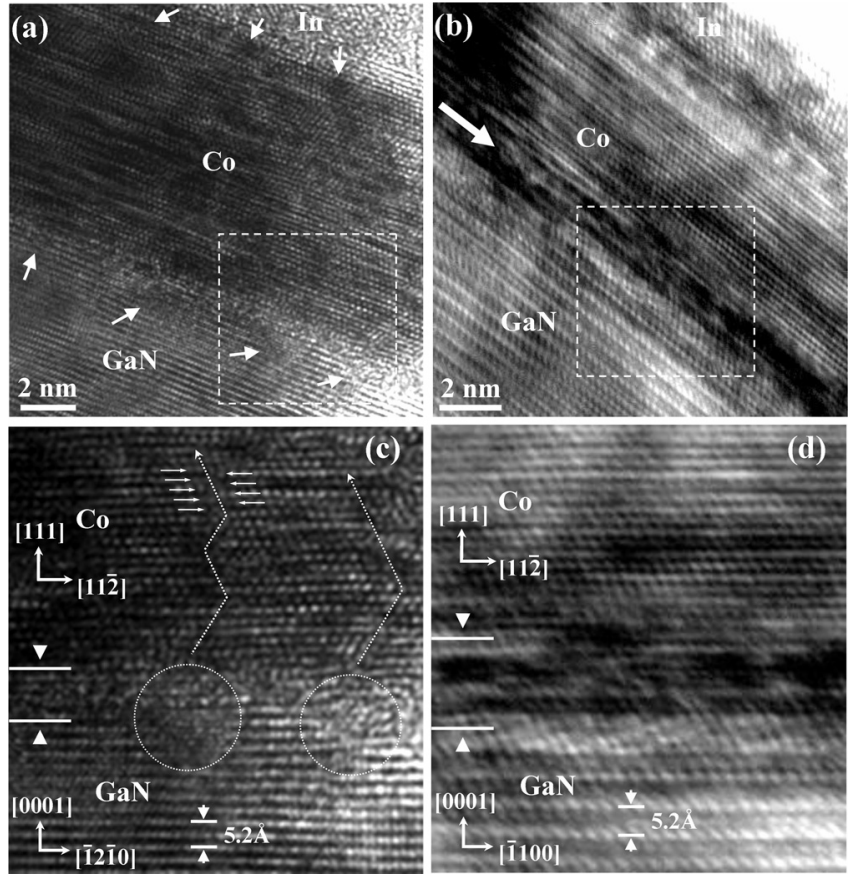

FIG. 3. HRTEM micrographs of (a) $\mathrm{Co} / \mathrm{GaN}(0001)-“ 1 \times 1$ " and (b) $\mathrm{Co} /$ GaN(0001)- $(1 \times 1)$ samples, respectively, after annealing. (c) and (d) show the zoom-in micrographs in the areas framed by the dashed boxes in (a) and (b), respectively.

happen during the fast cooling process, and so the hightemperature fcc phase is preserved. Note further that the zone axes of the TEM micrographs of Figs. 3(a) and 3(b) are along GaN [1010] and [1120], respectively. However, with respect to epitaxial Co, they are of the same [11 0 ] azimuth, in accordance with the earlier observations that $30^{\circ}$-rotated growth domains dominate Co growth on $\mathrm{GaN}(0001)-“ 1 \times 1$ " while aligned epitaxy is achieved on $\operatorname{GaN}(0001)-(1 \times 1){ }^{5}$ Secondly, apart from thin disordered, amorphous-like regions at the very interface of $\mathrm{Co} / \mathrm{GaN}$, the epitaxial $\mathrm{Co}$ films retain very good structural stability upon extensive annealing at $1000 \mathrm{~K}$. No structural phases related to cobalt gallides or cobalt nitrides are observed. Although the lattices of Co grown on $\mathrm{GaN}(0001)$-" $1 \times 1$ " is somewhat distorted, the film on $\mathrm{GaN}(0001)-(1 \times 1)$ shows quite good lattice coherence. The disordered, amorphous-like interface region appears thinner for $\mathrm{Co} / \mathrm{GaN}(0001)-" 1 \times 1$ " than in $\mathrm{Co} /$ $\mathrm{GaN}(0001)-(1 \times 1)$, though it seems less uniform with voids and protrusions distributing along the interface region [examples are indicated in Fig. 3(a) by arrows at bottom and circled in the enlarged image of Fig. 3(c)]. At these locations, extended defects such as partial dislocations and stacking faults originate and slip along zigzag paths alternating between directions making an angle of $\sim 109^{\circ}$ as marked by the zigzag dashed arrows in Fig. 3(c), which correspond nicely to the intersecting angle between fcc planes. Further, these paths penetrate through the whole film and end at the surface [downward arrows in Fig. 3(a)]. It is such defects that bring about the submonolayer high misalignment of the crystal planes, as indicated by the array of horizontal solid arrows in Fig. 3(c). In comparison, though the amorphous interface layer is thicker between $\mathrm{Co}$ and $\mathrm{GaN}(0001)$ - 


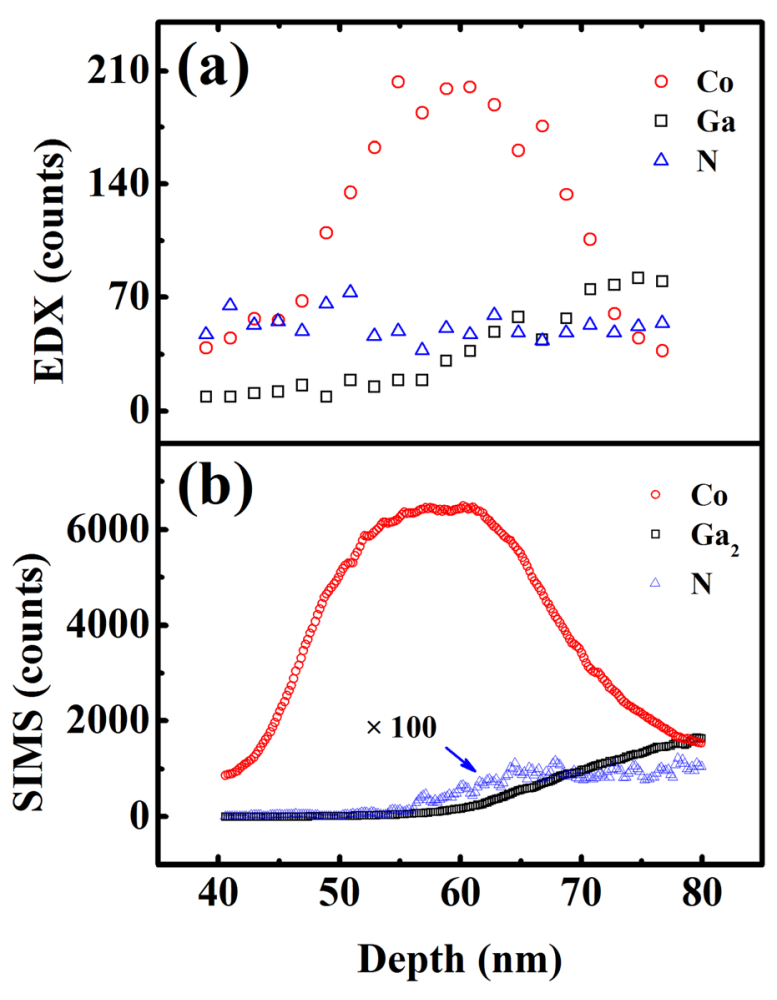

FIG. 4. (Color online) (a) EDX data measured at different locations of thickness of the cross-section $\mathrm{Co} / \mathrm{GaN}$-" $1 \times 1$ " TEM sample, while (b) is the SIMS depth profile of the sample showing the distribution of $\mathrm{Co}, \mathrm{Ga}$, and $\mathrm{N}$ atoms in the epitaxial Co film.

$(1 \times 1)$, it appears more uniform [Figs. 3(b) and 3(d)], associated with which is a low density of extended defects.

To examine the chemical composition of the epifilms and the hetero-interfaces, EDX and SIMS depth profile measurements were carried out. Figures 4(a) and 4(b) present, respectively, the EDX and SIMS results from a 10 nm-thick Co-on-GaN(0001)-“ $1 \times 1$ " sample, which had been capped by a $40 \mathrm{~nm}$-thick indium layer before being taken out of UHV for measurements (therefore, the profiles of Fig. 4 starts from $40 \mathrm{~nm}$ ). It can be found that both $\mathrm{Ga}$ and $\mathrm{N}$ are detectable in the Co film, though the Ga concentration appears decreasing with film thickness toward the surface, whereas $\mathrm{N}$ shows more or less a constant density. It thus seems that there is an intermixing between $\mathrm{Co}$ and $\mathrm{GaN}$. As mentioned earlier, no structural phases related to $\mathrm{Co}-\mathrm{Ga}$ and/or $\mathrm{Co}-\mathrm{N}$ compounds were found by TEM, so the $\mathrm{Ga}$ and $\mathrm{N}$ atoms from the substrate likely form dilute solutions in epitaxial Co.

\section{SUMMARY AND CONCLUSION}

Growth and structural properties of epitaxial Co on $\mathrm{GaN}(0001)-" 1 \times 1$ " and $(1 \times 1)$ surfaces have been studied and compared. As-deposited Co films, particularly those on $\mathrm{GaN}(0001)-(1 \times 1)$, show rougher surface morphologies than those after annealing. Examinations by STM and TEM reveal structural defects as well as complex interface structures in samples. In addition to rotation domains in epitaxial Co on GaN(0001)-" $1 \times 1$ ", stacking faults are abundant, giving rise to surface features of submonolayer high steps. On GaN(0001)-( $(1 \times 1)$, on the other hand, single domain crystalline films are achieved, which is accompanied by much fewer stacking defects. TEM examinations of the samples show the presence of disordered interface layers between Co and $\mathrm{GaN}$, where a relatively non-uniform disordered interface is likely the source of the extended defects in film. Compositional measurements indicate a mixing of $\mathrm{GaN}$ and $\mathrm{Co}$, probably in the form of dilute solution of $\mathrm{Ga}$ and/or $\mathrm{N}$ in Co. The structural defects in film will likely affect the magnetic properties of the FM films, ${ }^{5}$ while the interface state will affect spin injection from FM electrode to semiconductor in spintronic devices. It thus remains challenging to utilize $\mathrm{Co} /$ GaN heterostructures for spintronic applications until significant improvements are made toward crystal structural and interface integrity. These will form the central themes of future research in this heteroepitaxial system.

\section{ACKNOWLEDGMENTS}

We acknowledge technical support from Mr. W. K. Ho. This work was financially supported from the NSFC/RGC Joint Research Scheme of the Natural Science Foundation of China and Hong Kong Special Administrative Region, China, under Grant No. N_HKU705/07.

${ }^{1}$ C. A. F. Vaz, J. A. C. Bland, and G. Lauhoff, Rep. Prog. Phys. 71, 78 (2008).

${ }^{2}$ K. Ando, Science 3121883 (2006); D. Awschalom, N. Samarth, and D. Loss, Semiconductor Spintronics and Quantum Computation (Springer, New York, 2002).

${ }^{3}$ P. LeClair, J. T. Kohlhepp, C. H. van de Vin, H. Wieldraaijer, H. J. M. Swagten, W. J. M. de Jonge, A. H. Davis, J. M. MacLaren, J. S. Moodera, and R. Jansen, Phys. Rev. Lett. 88, 107201 (2002); A. L. Vázquez de Parga, F. J. García-Vidal, and R. Miranda, Phys. Rev. Lett. 85, 4365 (2000).

${ }^{4}$ V. M. Bermudez, R. Kaplan, M. A. Khan, and J. N. Kuznia, Phys. Rev. B 48, 2436 (1993).

${ }^{5}$ H. D. Li, K. He, M. H. Xie, N. Wang, J. F. Jia, and Q. K. Xue, New J. Phys. 12, 073007 (2010).

${ }^{6}$ A. R. Smith, R. M. Feenstra, D. W. Greve, M. S. Shin, M. Skowronski, J. Neugebauer, and J. E. Northrup, Appl. Phys. Lett. 72, 2114 (1998); A. R. Smith, R. M. Feenstra, D. W. Greve, M. S. Shin, M. Skowronski, J. Neugebauer, and J. E. Northrup, Surf. Sci. 423, 70 (1999); S. H. Xu, H. Wu, X. Q. Dai, W. P. Lau, L. X. Zheng, M. H. Xie, and S. Y. Tong, Phys. Rev. B 67, 125409 (2003).

${ }^{7}$ J. E. Northrup, J. Neugebauer, R. M. Feenstra, and A. R. Smith, Phys. Rev. B 61, 9932 (2000).

${ }^{8}$ Z. X. Yu, S. Y. Tong, S. H. Xu, S. Ma, and H. S. Wu, Surf. Rev. Lett. 10, 831 (2003).

${ }^{9}$ M. H. Xie, S. M. Seutter, W. K. Zhu, L. X. Zheng, H. Wu, and S. Y. Tong, Phys. Rev. Lett. 822749 (1999).

${ }^{10}$ E. Lundgren, B. Stanka, M. Schmid, and P. Varga, Phys. Rev. B 62, 2843 (2000).

${ }^{11}$ P. Tolédano, G. Krexner, M. Prem, H. P. Weber, and V. P. Dmitriev, Phys. Rev. B 64, 144104 (2001). 